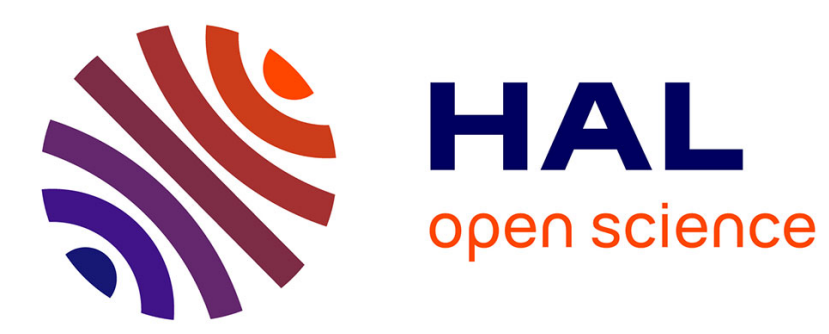

\title{
Switched Observer Design For a Class of Locally Unobservable Time-Varying Systems
}

Stanislav Aranovskiy, Denis Efimov, Dmitry Sokolov, Jian Wang, Igor Ryadchikov, Alexey Bobtsov

\section{- To cite this version:}

Stanislav Aranovskiy, Denis Efimov, Dmitry Sokolov, Jian Wang, Igor Ryadchikov, et al.. Switched Observer Design For a Class of Locally Unobservable Time-Varying Systems. Automatica, 2021, 130, pp.109715. 10.1016/j.automatica.2021.109715 . hal-03273675

\section{HAL Id: hal-03273675 \\ https://hal.science/hal-03273675}

Submitted on 29 Jun 2021

HAL is a multi-disciplinary open access archive for the deposit and dissemination of scientific research documents, whether they are published or not. The documents may come from teaching and research institutions in France or abroad, or from public or private research centers.
L'archive ouverte pluridisciplinaire HAL, est destinée au dépôt et à la diffusion de documents scientifiques de niveau recherche, publiés ou non, émanant des établissements d'enseignement et de recherche français ou étrangers, des laboratoires publics ou privés. 


\title{
Switched Observer Design For a Class of Locally Unobservable Time-Varying Systems
}

\author{
Stanislav Aranovskiy ${ }^{\mathrm{a}, \mathrm{b}}$, Denis Efimov $^{\mathrm{c}}$, Dmitry Sokolov $^{\mathrm{d}}$, Jian Wang ${ }^{\mathrm{e}}$, \\ Igor Ryadchikov ${ }^{\mathrm{f}}$, Alexey Bobtsov ${ }^{\mathrm{b}}$ \\ ${ }^{a}$ IETR - CentaleSupélec, Avenue de la Boulaie, 35576 Cesson-Sévigné, France \\ ${ }^{\mathrm{b}}$ Faculty of Control Systems and Robotics, ITMO University, 197101 Saint Petersburg, Russia \\ ${ }^{\mathrm{c}}$ Univ. Lille, Inria, CNRS, UMR 9189 - CRIStAL, F-59000 Lille, France. \\ ${ }^{\mathrm{d}}$ Université de Lorraine, CNRS, Inria, LORIA, F-54000 Nancy, France \\ ${ }^{\mathrm{e}}$ School of Automation, Hangzhou Dianzi University, Xiasha Higher Education Zone, Hangzhou, Zhejiang, PR China \\ ${ }^{\mathrm{f}}$ Kuban State University, Krasnodar, Russia
}

\begin{abstract}
We consider the problem of state estimation for a parameter-varying system that is unobservable for some values of timevarying parameters. The set of parameter values is divided into a finite number of subsets for which the system is observable, and a switched observer is proposed. A dwell-time condition is defined that ensures exponential convergence. The conditions of stability are formulated in the form of matrix inequalities, which can be used for gains tuning. The obtained theoretical results are supported with an illustrative example and experimental studies for a reaction-wheel pendulum testbench.
\end{abstract}

Key words: parameter-varying systems, switched observer, switched Lyapunov function, dwell-time

\section{Introduction}

State estimation for time-varying systems is a longstanding problem that has numerous engineering applications [20]. First solutions to this problem based on Kalman filtering were proposed in 1960s [10], however, this problem is not entirely solved and remains an active research topic. In [5] it has been shown that for a class of nonlinear systems the state estimation problem can be translated (via a change of coordinates) to the state estimation problem for a linear time-varying system, and an adaptive observer has been applied. Another adaptive observer for time-varying systems with parametric un-

\footnotetext{
* Corresponding author J. Wang.

Email addresses:

stanislav. aranovskiy@centralesupelec.fr (Stanislav Aranovskiy), denis.efimov@inria.fr (Denis Efimov), dmitry.sokolov@univ-lorraine.fr (Dmitry Sokolov), wangjian119@hdu.edu.cn (Jian Wang), ryadchikov@kubsu.ru (Igor Ryadchikov), bobtsov@mail.ifmo.ru (Alexey Bobtsov).
}

certainties has been reported in [29], which is also applicable to a class of state-affine nonlinear systems. Setmembership estimation and interval observers for timevarying systems have been proposed in [14,11], and estimation with finite and fixed-time for a class of timevarying system has been studied in [23]; see [7] and references therein for more details on observer design. However, these approaches typically assume that the estimated system is observable, or, more precisely, uniformly completely observable $[7,15]$. This assumption can be violated in some applications, e.g., in power converters [18], where a particular combination of input signals or time-varying parameters may make the plant unobservable, at least for some instances of time. Moreover, it is possible that for some combinations of input parameters, the system dynamics is changed significantly, and a single observer with constant parameters is not capable of tracking the states in different operation modes. This problem can be addressed by time-varying observers, where we are interested in the particular class of switched observers. 
Switched observers are typically applied for state estimation in processes with commutations of dynamics, where the system can operate in a finite number of operation modes. Whereas there exist nonlinear observers for nonlinear switched systems [18,27], linear switched system state estimation is commonly addressed through linear switched observers. The conventional approach is to construct a common Lyapunov function that is suitable for all operation modes, such as [2], which has been also improved in [1] by applying a projection method. The key tools for this approach are Linear Matrix Inequalities (LMI), for which powerful numerical solvers are available [19]. However, the existence of a common Lyapunov function is a restrictive assumption, and, particularly, it does not hold if some operation modes are not observable. This problem has been addressed in [28], where authors proposed conditions under which the system is observable even if some individual modes are unobservable. The same problem has been considered in [17], where the authors studied when does there exist a trajectory making the system observable.

The common Lyapunov function requirement can be relaxed by imposing assumptions on the Average Dwell Time (ADT) of commutation, as in $[21,6]$. The ADT concept can also be used when the switching signal is not precisely known or is measured with a delay, as in [30]. Another interesting solution has been proposed in [22], where the switching signal is not available and has to be estimated. To this end, a switched observer has been constructed in such a way that it ensures boundedness of estimation error if the switching signal is unknown, and it guarantees convergence when the operation mode is identified correctly. It is worth noting that for some observers, e.g., [6,22], switches in operation modes can yield jumps in estimates, i.e., observer's states, that makes these estimators rather hybrid than switched.

Novelty and Contribution. ${ }^{1}$ In this paper, we consider a class of linear time-varying (or parameter-varying) systems, which are not observable for certain values of the varying parameters. Considered systems are not switched themselves and do not have a finite number of operation modes. However, for the considered class of systems, we assume that the set of parameter values is divided into a finite number of subsets, where for each subset the system is observable, and that the observability is lost only when the vector of varying parameters travels from one subset to another. Motivated by this assumption, we propose a novel switched observer and show that its parameters can be designed as a solution to an LMI problem. We also derive the dwell-time conditions imposed on the switching signals to establish and estimate the exponential convergence

\footnotetext{
1 This paper extends the preliminary results reported in [3]. Compared to [3], this work considers the general case of $n$-order systems, contains detailed proofs and experimental results.
}

rate. Moreover, we show that it is sufficient to solve the LMI for one set of parameters only, and other gains can be computed from the obtained solution; this property significantly simplifies the design procedure.

The rest of the paper is organized as follows. In Section 2, we present an illustrative example to motivate the considered problem and illustrate that some straightforward solutions do not apply. The formal problem statement is given in Section 3, and the main result is presented in Section 4. An illustrative observer design example is given in Section 5, and results of experimental studies for a reaction wheel pendulum are reported in Section 6 . The conclusion of the paper is in Section 7. Finally, some technical details of proofs can be found in the Appendix.

Used notations. For integers $n, m$, we define $I_{n}$ as the $n \times n$ unit matrix, and $0_{n \times m}$ and $1_{n \times m}$ as $n \times m$ matrices of zeros and ones, respectively.

\section{Motivating example}

As a motivating example, we consider the reaction-wheel pendulum testbench that was also presented in [26], see Section 6 for more details. Neglecting the friction, the pendulum dynamics can be written as

$$
\ddot{\theta}(t)=a \sin (\theta(t))+b I(t)
$$

where $a>0$ and $b>0$ are constants depending on the system's parameters, $\theta$ is the pendulum angle, where the upward position is chosen as zero, and $I$ is the current in the motor windings. Assuming an internal fast-timescale current regulation, we consider $I(t)=u(t)$, where $u$ is our input control signal. The observer goal is to estimate the pendulum velocity $\dot{\theta}$. However, due to possible imprecisions in sensor placement and pendulum alignment, the zero reading of the sensor does not coincide with the equilibrium position, and the angle $\theta$ is measured with a certain constant offset, i.e., the measured signal $y$ is given by

$$
y(t)=\theta(t)+d
$$

where $d$ is the constant bias.

Assuming that the bias is sufficiently small and approximating $\sin (\theta)=\sin (y-d) \approx \sin (y)-\cos (y) d$, the problem of the velocity $\dot{\theta}$ and the bias $d$ estimation can be formulated as the state estimation problem of the system $^{2}$

$$
\dot{x}=A(y) x+\beta(u, y), y=C x,
$$

\footnotetext{
2 When clear from the context, in the sequel the argument of time is omitted.
} 
where $x:=[\theta+d, \dot{\theta},-a d]^{\top}$

$$
A(y):=\left[\begin{array}{ccc}
0 & 1 & 0 \\
0 & 0 & \cos (y) \\
0 & 0 & 0
\end{array}\right], C:=\left[\begin{array}{lll}
1 & 0 & 0
\end{array}\right]
$$

and $\beta(u, y):=[0, a \sin (y)+b u, 0]^{\top}$. We also assume that the input signal $u$ is such that trajectories of the system (2) exist and are unique for all $t \geq 0$.

It is worth noting that the observability matrix of the system (2) is given by

$$
\mathcal{O}=\left[\begin{array}{ccc}
1 & 0 & 1 \\
0 & 1 & 0 \\
0 & 0 & \cos (y)
\end{array}\right]
$$

and it is singular for $\cos (y)=0$. This obviously implies that some constrains should be imposed for the signal $y, e . g$., the states $x_{1}$ and $x_{3}$ cannot be reconstructed for $y(t) \equiv \pm \frac{\pi}{2}$. To simplify the example, assume that $y \in \Omega_{y}:=\left\{y \mid \cos ^{2}(y) \geq \delta_{y}^{2}\right\}$ for some $\delta_{y} \in(0,1)$, i.e., the system is always observable but $\cos (y)$ may change its sign at some isolated instants of time.

One classic solution for linear time-varying systems is to construct a linear time-varying observer in the form

$$
\dot{\hat{x}}=A(y) \hat{x}+\beta(u, y)-H C^{\top}(C \hat{x}-y),
$$

where the time-varying symmetric gain matrix $H(t) \in$ $\mathbb{R}^{3 \times 3}$ is the solution of the matrix differential equation

$$
\dot{H}=H A^{\top}(y)+A(y) H-H C^{\top} C H+Q
$$

for some $H(0)=H_{0}>0$, and $Q>0$ is the design parameter. It is known (see, e.g., $[25,24]$ ) that the observer (4) ensures exponential convergence if the system is uniformly observable, that is there exist $T_{O}, \delta_{1}$, and $\delta_{2}$, all positive, such that for all $t$

$$
\delta_{1} I_{3} \leq \int_{t}^{t+T_{O}} \Phi^{\top}(\tau, t) C^{\top} C \Phi(\tau, t) d \tau \leq \delta_{2} I_{3},
$$

where $\Phi(\cdot, \cdot)$ is the state-transition matrix of the system (2). The uniform observability can be connected with the assumption that the system (2) does not stuck in the domain where $\cos (y) \approx 0$. However, the implementation of the observer (4) in embedded systems has certain drawbacks since computation of the gain matrix $H(t)$ requires to solve online 6 differential equations with quadratic terms that may be sensitive to numerical methods. Thus, in what follows we aim to designs that are less demanding for online computations than the observer (4), e.g., by the means of off-line gains precalculation.

Let us show that some straightforward solutions do not apply to this problem. To this end, consider the observer

$$
\dot{\hat{x}}=A(y) \hat{x}+\beta(u, y)-L(y)(C \hat{x}-y),
$$

where the parameter-varying gain vector $L(y)$ is to be defined, and $\hat{x}$ is the estimate of $x$. Define the estimation error as $e:=\hat{x}-x$, then

$$
\dot{e}=(A(y)-L(y) C) e .
$$

A simple solution would be to find a constant vector $L$ stabilizing the system for all values of $y \in \Omega_{y}, e . g$., with a common Lyapunov function; however, such a solution does not exist. Indeed, if $L$ is stabilizing for all $y$, then for any $y \in \Omega_{y}$ the matrix $A(y)-L C$ should be Hurwitz. The characteristic polynomial of the error state matrix is

$$
s^{3}+s^{2}\left(l_{1}+l_{3}\right)+s l_{2}+\cos (y) l_{3},
$$

where $l_{i}$ is the $i$-th element of the vector $L$, and the error dynamics cannot be stabilized for positive and negative values of $\cos (y)$ with the same gain $l_{3}$.

Hence, we have to calculate a vector function $L(y)$. Methods of design of LPV observers typically consider the quadratic Lyapunov function $V=e^{\top} P e$, where the matrix $P$ can be constant or parameter-varying, $P=P(y)$. The main drawback of the (continuous in $y$ ) parameter-varying matrix $P(y)$ is that the time derivative of the Lyapunov function will depend on the time derivative of the signal $y$ implying some probably restrictive assumptions on boundedness of $\dot{y}$. Concerning the constant matrix $P$, it can be shown that the system (2) does not admit such a solution, or more precisely, there do not exist a parameter-varying gain vector $L(y)$ and a positive-definite constant matrix $P$, such that for all $y \in \Omega_{y}$ the linear matrix inequality (LMI)

$$
(A(y)-L(y) C)^{\top} P+P(A(y)-L(y) C)<0
$$

holds, see Proposition 7 in Appendix.

Motivated by the discussed shortcomings, we propose to exploit the canonical form of the system (2) and design a switched observer. For this observer, we consider the Lyapunov function $V=e^{\top} P(y) e$, where $P(y)$ is piecewise constant. Thus, we do not impose assumption on the time derivative of $y$ but study possible jumps of the value of the Lyapunov function when switches occur. In next section, we consider a more general problem statement, for which the system (2) is a particular case. 


\section{Problem statement}

Consider an LPV SISO system in the following form

$$
\dot{x}=A(q) x+\beta(u, y, t), y=C x,
$$

where $x \in \mathbb{R}^{n}$ is the state, $u \in \mathbb{R}$ is the known input, $y \in \mathbb{R}$ is the measured output signal,

$$
A(q):=\left[\begin{array}{ccccc}
0 & q_{1} & 0 & \cdots & 0 \\
0 & 0 & q_{2} & \cdots & 0 \\
\vdots & \vdots & \vdots & \ddots & \vdots \\
0 & 0 & 0 & \cdots & q_{n-1} \\
0 & 0 & 0 & \cdots & 0
\end{array}\right], C:=\left[\begin{array}{lllll}
1 & 0 & 0 & \cdots & 0
\end{array}\right]
$$

with $q=\left(q_{1}, q_{2}, \cdots, q_{n-1}\right), q_{i} \in[-1,1], i \in \bar{i}:=$ $\{1, \ldots, n-1\}$, being a known piecewise continuous timevarying signal. The function $\beta$ is known. We also assume that the input signal $u$ is such that trajectories of the system (5) exist and are unique for all $t \geq 0$. The goal is to design an observer for $x$.

The main issue for solution of this problem is that the system looses its observability for zero values of varying parameters $q_{1}, \ldots, q_{n-1}$. Indeed, the observability matrix of the system is

$$
\mathcal{O}=\left[\begin{array}{ccccc}
1 & 0 & 0 & \cdots & 0 \\
0 & q_{1} & 0 & \cdots & 0 \\
0 & 0 & q_{1} q_{2} & \cdots & 0 \\
\vdots & \vdots & \vdots & \ddots & \vdots \\
0 & 0 & 0 & \cdots & \prod_{i=1}^{n-1} q_{i}
\end{array}\right]
$$

As it can be seen from the observability matrix, the system is observable when all elements of $q$ are not zero, the rank of the observability matrix equals $n-1$ and the state $x_{n}$ is not observable when the only zero element of $q$ is $q_{n-1}$, and for $q_{1}=0$ only the state $x_{1}$ is observable. Thus, a hypothesis has to be introduced that the system does not spend much time in the mode where any element of $q$ equals zero. This hypothesis will be formulated as a sufficient condition for the signal $q$ ensuring observer convergence.

Remark 1 Since the input $\beta(u, y, t)$ is known, it can be canceled in the observer design and thus it does not impact neither the error dynamics nor the stability analysis. However, it allows us considering a wider class of systems with known inputs, e.g., the mechanical system example in Section 2.

Remark 2 In [3] it was shown that for $n=3$ the state estimation problem can be solved with a nonlinear adap- tive observer. To this end, an instrumental output signal was constructed via a nonlinear transformation, and a gradient-like update law was proposed. The exponential convergence was shown under assumptions on the trajectory in the vein of the persistency of excitation property. However, the instrumental signal construction was proposed only for $n=3$, and its extension to a more general case $n>3$ can be complicated. Thus, we do not consider that approach in this paper in detail.

\section{Main result}

\subsection{Observer design}

To present our solution, note that the vector $q$ belongs to the hypercube in $\mathbb{R}^{n-1}$ that has $2^{n-1}$ vertices of the form $( \pm 1, \pm 1, \cdots, \pm 1)$. Let us enumerate these vertices in any particular order, $v^{k}, k \in \bar{k}:=\left\{1,2, \ldots, 2^{n-1}\right\}$. Let us subdivide the hypercube into $2^{n-1}$ closed smaller hypercubes $Q_{k}$. These hypercubes cover the original hypercube, and their interiors are disjoint. For each $k$, the corresponding smaller hypercube $Q_{k}$ is uniquely defined by two opposite vertices: the origin and the point $v^{k}$. For example, for $n=3, \bar{i}=\{1,2\}$, and $\bar{k}=\{1,2,3,4\}$, one possible enumeration is

$$
\begin{aligned}
& v^{1}:=(1,1), v^{2}:=(1,-1), \\
& v^{3}:=(-1,-1), v^{4}:=(-1,1), \\
& \mathcal{Q}_{1}:=\left\{\left(q_{1}, q_{2}\right) \mid 1 \geq q_{1} \geq 0,1 \geq q_{2} \geq 0\right\}, \\
& \mathcal{Q}_{2}:=\left\{\left(q_{1}, q_{2}\right) \mid 1 \geq q_{1} \geq 0,-1 \leq q_{2} \leq 0\right\}, \\
& \mathcal{Q}_{3}:=\left\{\left(q_{1}, q_{2}\right) \mid-1 \leq q_{1} \leq 0,-1 \leq q_{2} \leq 0\right\}, \\
& \mathcal{Q}_{4}:=\left\{\left(q_{1}, q_{2}\right) \mid-1 \leq q_{1} \leq 0,1 \geq q_{2} \geq 0\right\} .
\end{aligned}
$$

Any point of the trajectory $q$ belongs to exactly one of the hypercubes, with an exception of the trajectory crossing the boundary between the hypercubes. For those points that belong to several hypercubes simultaneously, the notation $q \in \mathcal{Q}_{k}$ implies that the smaller index $k$ among the considered hypercubes is chosen.

Define the sign function as

$$
\operatorname{sgn}\left(q_{i}\right):=\left\{\begin{array}{l}
1 \text { if } q_{i} \geq 0 \\
-1 \text { if } q_{i}<0
\end{array}\right.
$$

and the set of $2^{n-1}$ matrices

$$
A_{k}:=\left[\begin{array}{ccccc}
0 & \operatorname{sgn}\left(v_{1}^{k}\right) & 0 & \cdots & 0 \\
0 & 0 & \operatorname{sgn}\left(v_{2}^{k}\right) & \cdots & 0 \\
\vdots & \vdots & \vdots & \ddots & \vdots \\
0 & 0 & 0 & \cdots & \operatorname{sgn}\left(v_{n-1}^{k}\right) \\
0 & 0 & 0 & \cdots & 0
\end{array}\right]
$$


In other words, the matrix $A_{k}$ has the same structure as the matrix $A(q)$ where the parameters $q_{1}, \ldots, q_{n-1}$ are replaced by their extremes in the set $\mathcal{Q}_{k}, k \in \bar{k}$, and the matrices $A_{k}$ form a convex polytope where the matrix function $A(q)$ is embedded. Note that all pairs $\left(C, A_{k}\right)$ are observable.

Define the function $f(q, x): \mathbb{R}^{n-1} \times \mathbb{R}^{n} \rightarrow \mathbb{R}^{n-1}$, where the $i$-th component is

$$
f_{i}(q, x)=\left(q_{i}-\operatorname{sgn}\left(q_{i}\right)\right) x_{i+1},
$$

and consider the switched observer

$$
\begin{aligned}
\dot{\hat{x}} & =A_{s}(q) \hat{x}+\beta(u, y, t)+B f(q, \hat{x}) \\
& -L_{s}(q)(C \hat{x}-y),
\end{aligned}
$$

where $A_{s}(q)$ is the switched matrix, $A_{s}(q)=A_{k}$ if $q \in$ $\mathcal{Q}_{k}$,

$$
B:=\left[\begin{array}{c}
I_{n-1} \\
0_{1 \times(n-1)}
\end{array}\right],
$$

and $L_{s}(q)$ is the switched gain defined as $L_{s}(q):=L_{k}$ when $q \in \mathcal{Q}_{k}, k \in \bar{k}$, and the gains $L_{k}$ are computed as

$$
L_{k}=\mathcal{O}_{k}^{-1} \mathcal{O}_{1} L_{1}
$$

where $\mathcal{O}_{k}$ is the observability matrix of the pair $\left(C, A_{k}\right)$ and $L_{1}$ is to be defined.

In what follows, we show that if a certain LMI is feasible, then there exists $L_{1}$ such that under some assumptions on the signal $q$ the observer (7) ensures exponential convergence of the estimate $\hat{x}$ to the state vector $x$ of the system (5). To streamline the presentation and to gradually introduce all required definitions, we first present analysis of the estimation error dynamics, and then summarize it in Theorem 4.

\subsection{Convergence analysis}

With the definitions in use, we can rewrite the system (5) as

$$
\dot{x}=A_{s}(q) x+\beta(u, y, t)+B f(q, x) .
$$

Then the error dynamics is given by

$$
\dot{e}=\left(A_{s}(q)-L_{s}(q) C\right) e+B \Delta f,
$$

where $e=\hat{x}-x$ and $\Delta f:=f(q, \hat{x})-f(q, x)$. Then for $\Delta f$ it holds

$$
\Delta f_{i}^{2}=e_{i+1}^{2}-\gamma\left(q_{i}\right) e_{i+1}^{2},
$$

where the function $\gamma: \mathbb{R} \rightarrow \mathbb{R}$ is defined as

$$
\gamma\left(q_{i}\right):=\left|q_{i}\right|\left(2-\left|q_{i}\right|\right) .
$$

Note that $\gamma(0)=0, \gamma(1)=1$, and $\gamma\left(q_{i}\right)$ is monotonically increasing as $\left|q_{i}\right|$ varies from 0 to 1 .

Consider first the case $q \in \mathcal{Q}_{1}$ and the function $V_{1}(e):=$ $e^{\top} P_{1} e$, where the matrix $P_{1}=P_{1}^{\top}>0$. The time derivative of the function $V_{1}$ along trajectories of $e$ is

$$
\begin{aligned}
\dot{V}_{1} & =e^{\top}\left(\left(A_{1}-L_{1} C\right)^{\top} P_{1}+P_{1}\left(A_{1}-L_{1} C\right)\right) e \\
& +2 e^{\top} P_{1} B \Delta f+\Delta f^{\top} \Delta f-\Delta f^{\top} \Delta f .
\end{aligned}
$$

Choose $\delta_{q} \in(0,1)$ and compute $\delta_{\gamma}:=\gamma\left(\delta_{q}\right), 0<\delta_{\gamma}<1$. Then by adding and subtracting the term $e_{1}^{2}-\delta_{\gamma}|e|^{2}$ and recalling that due to $(9)$

$$
\Delta f^{\top} \Delta f=\sum_{i=1}^{n-1}\left(e_{i+1}^{2}-\gamma\left(q_{i}\right) e_{i+1}^{2}\right),
$$

one obtains

$$
\dot{V}_{1}=\left[\begin{array}{ll}
e^{\top} & \Delta f^{\top}
\end{array}\right] M_{1}\left[\begin{array}{c}
e \\
\Delta f
\end{array}\right]-e^{\top} Q(q) e,
$$

where

$$
\begin{gathered}
Q(q):=\left[\begin{array}{cccc}
1-\delta_{\gamma} & 0 & \cdots & 0 \\
0 & \gamma\left(q_{1}\right)-\delta_{\gamma} & \cdots & 0 \\
\vdots & \vdots & \ddots & \vdots \\
0 & 0 & 0 & \gamma\left(q_{n-1}\right)-\delta_{\gamma}
\end{array}\right], \\
M_{1}:=\left[\begin{array}{ccc}
F_{1}+\left(1-\delta_{\gamma}\right) I_{n} & P_{1} B \\
B^{\top} P_{1} & & -I_{n-1}
\end{array}\right],
\end{gathered}
$$

and

$$
F_{1}:=\left(A_{1}-L_{1} C\right)^{\top} P_{1}+P_{1}\left(A_{1}-L_{1} C\right) .
$$

Let $P_{1}$ and $L_{1}$ be a solution of the matrix inequality $M_{1} \leq 0$ for some $\delta_{\gamma}$. Then

$$
\dot{V}_{1} \leq-e^{\top} Q(q) e \leq-\frac{\gamma\left(\min _{j \in \bar{i}}\left|q_{j}\right|\right)-\delta_{\gamma}}{\lambda_{M}} V_{1},
$$

where $\lambda_{M}$ is the maximum eigenvalue of $P_{1}$.

Consider now the case $q \in \mathcal{Q}_{k}$ for $k \neq 1$ and the function $V_{k}(e)=e^{\top} P_{k} e$, where $P_{k}=P_{k}^{\top}>0$. Repeating the same steps as above, we obtain

$$
\dot{V}_{k}=\left[\begin{array}{ll}
e^{\top} & \Delta f^{\top}
\end{array}\right] M_{k}\left[\begin{array}{c}
e \\
\Delta f
\end{array}\right]-e^{\top} Q(q) e,
$$


where

$$
M_{k}:=\left[\begin{array}{cc}
F_{k}+\left(1-\delta_{\gamma}\right) I_{n} & P_{k} B \\
B^{\top} P_{k} & -I_{n-1}
\end{array}\right],
$$

and

$$
F_{k}:=\left(A_{k}-L_{k} C\right)^{\top} P_{k}+P_{k}\left(A_{k}-L_{k} C\right) .
$$

Now we will show that if there exist $L_{1}$ and $P_{1}$ such that $M_{1} \leq 0$, then there exist also $P_{k}$ and $L_{k}$ such that $M_{k} \leq 0$. Recalling that $\mathcal{O}_{k}$ is the observability matrix of the pair $\left(A_{k}, C\right)$, define the matrices

$$
R_{k}:=\mathcal{O}_{k}^{-1} \mathcal{O}_{1}
$$

for $k \in \bar{k}$. Due to the structure of the matrices $A$ and $C$, for any $k$ the matrix $R_{k}$ is diagonal and all its elements are either 1 or -1 . Thus, $R_{k}$ is a unitary matrix and $R_{k}=R_{k}^{-1}$. Moreover, it holds

$$
A_{k}=R_{k} A_{1} R_{k}, C R_{k}=C
$$

Choose

$$
P_{k}=R_{k} P_{1} R_{k}, L_{k}=R_{k} L_{1}
$$

Then

$$
M_{k}=\left[\begin{array}{cc}
R_{k} & 0 \\
0 & I_{n-1}
\end{array}\right] M_{k}^{\prime}\left[\begin{array}{cc}
R_{k} & 0 \\
0 & I_{n-1}
\end{array}\right]
$$

where

$$
M_{k}^{\prime}=\left[\begin{array}{cc}
F_{1}+\left(1-\delta_{\gamma}\right) I_{n} & P_{1} R_{k} B \\
B^{\top} R_{k} P_{1} & -I_{n-1}
\end{array}\right]
$$

and $F_{1}$ is defined in (10). Due to the structure of the matrix $B$, the matrix $B B^{\top}$ is a diagonal matrix, thus

$$
P_{1} R_{k} B B^{\top} R_{k} P_{1}=P_{1} B B^{\top} P_{1}
$$

and the matrices $M_{1}$ and $M_{k}^{\prime}$ have the same Schur complement (see [13]) of the upper-left block element. Then the implication $M_{1} \leq 0 \Leftrightarrow M_{k}^{\prime} \leq 0$ holds and the choice (11) ensures $M_{k} \leq 0$.

Note that due to (11), the matrices $P_{k}$ have the same eigenvalues for all $k$. Therefore, for all $k \in \bar{k}$ and all fixed values of the switched matrix $A_{s}(q)$, for the switched dynamics we have

$$
\begin{aligned}
\dot{V}_{k} & \leq-e^{\top} Q(q) e \\
& \leq-\frac{\gamma\left(\min _{i \in \bar{i}}\left|q_{i}\right|\right)-\delta_{\gamma}}{\lambda_{M}} V_{k}=\left(\eta_{0}-\eta(q)\right) V_{k}
\end{aligned}
$$

where $\eta(q):=\frac{\gamma\left(\min _{i \in \bar{i}}\left|q_{i}\right|\right)}{\lambda_{M}}$ and $\eta_{0}:=\frac{\delta_{\gamma}}{\lambda_{M}}$.

Next we analyze possible jumps in the Lyapunov function values when switches occur. To this end, assume that the trajectory $q(t)$ crosses the border between the sets $\mathcal{Q}_{k}$ in isolated instants only. Define the Lyapunov function $V(e)=V_{k}(e)$ for $q \in \mathcal{Q}_{k}$ and let $t_{c}$ be the time instance when the commutation occurs and $q(t)$ travels from $\mathcal{Q}_{k}$ to $\mathcal{Q}_{j}$. Then the variation of the Lyapunov function admits the following upper estimate:

$$
|\Delta V|=\left|V\left(t_{c}^{+}\right)-V\left(t_{c}^{-}\right)\right|=\left|e^{\top}\left(P_{j}-P_{k}\right) e\right| \leq \mu V\left(t_{c}^{-}\right),
$$

for all $k, j \in \bar{k}$, where

$$
\mu:=\frac{\lambda_{M}}{\lambda_{m}}-1 \geq 0
$$

and $\lambda_{m}$ is the minimum eigenvalue of $P_{1}$. Note that a less conservative estimate of $\mu$ can be found computing the maximum generalized eigenvalue of the pair $P_{j}, P_{k}$ over all $j, k \in \bar{k}$.

It can be seen from (12) that the Lyapunov function decays when $\min _{i \in \bar{i}}\left|q_{i}\right|>\delta_{q}$, and may increase otherwise. To provide the exponential convergence, we must thus assume that the decrease of the Lyapunov function is in average (in the sense of an integral over a time interval) more important than the possible increase when $\min _{i \in \bar{i}}\left|q_{i}\right|<\delta_{q}$ or due to switches of $P_{k}$. It can be seen as a restriction that the trajectory $q(t)$ should not cross the borders between the sets $\mathcal{Q}$ too often and should not remain for a long time in small vicinities of the borders between these sets, where $\min _{i \in \bar{i}}\left|q_{i}\right|<\delta_{q}$ and the Lyapunov function may be increasing. More formally, this assumption can be formulated as follows.

Assumption 3 For the trajectory $q(t)$ there exist $T_{q}>$ $0, n_{\mathcal{Q}} \geq 0$, and $\kappa>0$ such that for all $t_{0} \geq 0$ during the time interval $\left[t_{0}, t_{0}+T_{q}\right]$ the trajectory $q(t)$ crosses the borders between the sets $\mathcal{Q}_{k}$ not more than $n_{\mathcal{Q}}$ times, and it holds

$$
\int_{t_{0}}^{t_{0}+T_{q}} \eta(q(\tau)) d \tau \geq n_{\mathcal{Q}} \ln (1+\mu)+\left(\eta_{0}+\kappa\right) T_{q} .
$$

If Assumption 3 holds, then for any $t_{0} \geq 0$ we have

$$
\begin{aligned}
V\left(t_{0}+T_{q}\right) & \leq V\left(t_{0}\right)(1+\mu)^{n_{\mathcal{Q}}} e^{\eta_{0} T_{q}} e^{-\int_{t_{0}}^{t_{0}+T_{q}} \eta(q(\tau)) d \tau} \\
& \leq e^{-\kappa T_{q}} V\left(t_{0}\right)<V\left(t_{0}\right)
\end{aligned}
$$

and $V(t)$ remains bounded for all $t \in\left[t_{0}, t_{0}+T_{q}\right]$,

$$
V(t) \leq V\left(t_{0}\right)(1+\mu)^{n_{\mathcal{Q}}} e^{\eta_{0} T_{q}}
$$

Then for all $t \geq 0$ it holds $V(t) \leq \Gamma e^{-\kappa t} V(0)$, where

$$
\Gamma:=(1+\mu)^{n_{\mathcal{Q}}} e^{\eta_{0} T_{q}} e^{\kappa T_{q}} .
$$


Thus, $V(t)$ is bounded and $V(t) \rightarrow 0$, where $\kappa$ is the exponential rate of convergence.

In Assumption 3, the inequality (14) depends not only on the trajectory $q(t)$ but also on the design parameters of the observer, i.e., on the eigenvalues $\lambda_{M}, \lambda_{m}$ of the matrix $P_{1}$. Thus, for the same trajectory $q(t)$ it is possible that Assumption 3 holds for one choice of observer parameters and does not hold for another. From this point of view, Assumption 3 may be considered as a convergence requirement for the designed observer.

Finally, applicability of the proposed switched observer is summarized in the following theorem, where the proof follows from the derivations above.

Theorem 4 Consider the system (5). Choose $\delta_{\gamma} \in$ $(0,1)$ such that there exist $P_{1}>0$ and $L_{1}$ satisfying the matrix inequality

$$
\left[\begin{array}{c|c}
\left(A_{1}-L_{1} C\right)^{\top} P_{1}+P_{1}\left(A_{1}-L_{1} C\right) & P_{1} B \\
+\left(1-\delta_{\gamma}\right) I_{n} & \\
\hline B^{\top} P_{1} & -I_{n-1}
\end{array}\right] \leq 0
$$

where $A_{1}=A\left(1_{(n-1) \times 1}\right)$, and $B$ is defined in (8). Then if the trajectory $q(t)$ satisfies Assumption 3 for $\eta(q)$ and $\eta_{0}$ defined in (12) and $\mu$ defined in (13), then the observer (7) ensures exponential convergence of the estimate $\hat{x}(t)$ to the state vector $x(t)$ of the system (5).

Remark 5 Assumption 3 can be relaxed by choosing $T_{q}$ and $\kappa$ non-uniformly in $t_{0}$ yielding asymptotic convergence instead of exponential.

Remark 6 Using standard methods for matrix inequalities, see [9], and defining $H_{1}:=L_{1}^{\top} P_{1}$, the matrix inequality (15) can be rewritten as the LMI

$$
\left[\begin{array}{c|c}
A_{1}^{\top} P_{1}+P_{1} A_{1}-C^{\top} H_{1}-H_{1}^{\top} C & P_{1} B \\
+\left(1-\delta_{\gamma}\right) I_{n} & \\
\hline B^{\top} P_{1} & -I_{n-1}
\end{array}\right] \leq 0,
$$

which can be efficiently solved for $P_{1}$ and $H_{1}$, see [19]. Then $L_{1}=P_{1}^{-1} H_{1}^{\top}$.

\section{Illustrative Example}

In this section, we continue the illustrative example started in Section 4.1. We consider the system (5) for $n=3$, where

$$
\beta(u, y, t):=-\left[\begin{array}{l}
3 \\
3 \\
1
\end{array}\right] y(t)+\left[\begin{array}{l}
0 \\
0 \\
1
\end{array}\right] \sin (2 \pi t)
$$

The trajectory $q=\left(q_{1}, q_{2}\right)$ is constructed as $q_{1}(t):=$ $\cos (t), q_{2}(t):=\cos (1.3 t)$, and the vertices $v^{k}$ and sets $\mathcal{Q}_{k}$, $k \in \vec{k}$, are chosen as (6). Choosing $\delta_{q}=0.05$ and computing $\delta_{\gamma} \approx 0.1$, a feasible solution to the matrix inequality (15) has been computed yielding $L_{1}=\left[\begin{array}{lll}74 & 451 & 69\end{array}\right]^{\top}$.

The state vector $x(t)$ and the estimation errors $e(t)$ are depicted in Fig. 1 and illustrate convergence of the state estimation errors. Fig. 2 shows the path of $q$, which is a Lissajous curve, and the Lyapunov function curve, where the level jumps can be observed at the switch instances, i.e., where $q_{1}(t)$ or $q_{2}(t)$ change their signs.

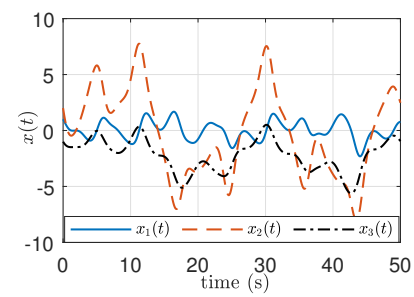

(a) State $x(t)$.

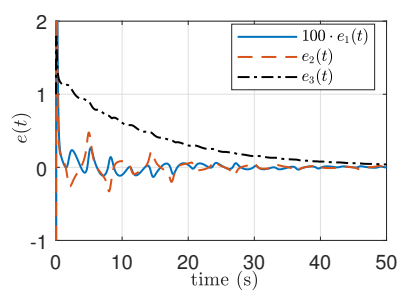

(b) Estimation error $e(t)$.
Fig. 1. State $x(t)$ of the plant and the estimation error $e(t)$ in the illustrative example.

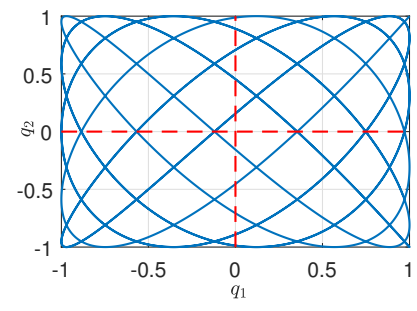

(a) The path $q(t)$ and the borders between the sets $\mathcal{Q}_{k}$.

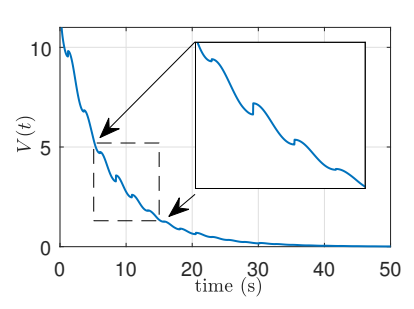

(b) The function $V(t)$.
Fig. 2. The path of $q$ and the Lyapunov function $V(t)$ in the illustrative example.

\section{State estimation for a mechanical system}

As described in Section 2, we consider the reactionwheel testbench that has been also presented in [26]. The pendulum hardware and the corresponding notation are given in Fig. 3, and the numerical values of parameters can be found in Table 1 of [26].
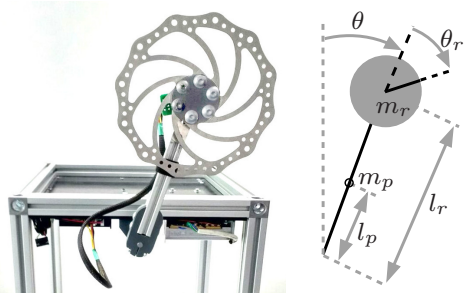

Fig. 3. Reaction-wheel pendulum hardware and the corresponding notation, see [26]. 
Neglecting the friction, the motion equations are

$$
\begin{aligned}
J_{r} \ddot{\theta}_{r}+J_{r} \ddot{\theta} & =k I, \\
\left(J+J_{r}\right) \ddot{\theta}+J_{r} \ddot{\theta}_{r} & =m l g \sin \theta,
\end{aligned}
$$

where $\theta_{r}$ and $\theta$ are the reaction wheel and the pendulum angles, respectively, $I$ is the current in the motor windings, $J, J_{r}, m l$, and $g$ are physical parameters, see [26]. From (16), the pendulum dynamics can be written as (1), where $a:=m l g / J$ and $b:=-k / J$. As discussed in Section 2, the angle $\theta$ is measured with a certain constant offset, $y(t)=\theta(t)+d$. It is well-known that such a bias affects stabilization performance and accuracy, see $[8,12]$. One possible solution is to estimate the bias constructing an extended observer. To this end, we recall that as discussed in Section 2, the velocity $\dot{\theta}$ and the bias $d$ can be estimated via observer design for the system (2). Note that the system (2) is in the form (5), where $q_{1}(t) \equiv 1$ and $q_{2}(t)=\cos (y(t))$, and thus the switched observer (7) can be constructed.

The proposed observer has been tested in the following scenario. First, an energy-based swing-up control strategy is applied as proposed in $[4,16]$. Second, when the pendulum is close to the upright position, the control law switches to the full-state feedback computed as optimal linear quadratic regulator (LQR), see [26]. In both phases, the pendulum velocity and the sensor bias are estimated with the proposed switched observer. The true value of the sensor bias is $d=5^{\circ} \approx 0.087$.

In the considered experiment, we chose the tuning values $\delta_{q}$ and $\delta_{\gamma}$ to be the same as in Section 5 , thus it yields the same gain vector $L_{1}=\left[\begin{array}{lll}74 & 451 & 69\end{array}\right]^{\top}$. The experimental results for $y(t)=\theta(t)+d$ and $\hat{d}(t)$ are depicted in Fig. 4 . The trajectory $y(t)$ starts in the bottom position, where $\theta(0)=\pi$, then after a series of swings it rises up and is stabilized at the upright position, where $\theta(t)=0$ and $y(t)=d$ in the steady state. The estimate of the bias converges to the true value. It is worth noting that during the swings, the observer switches multiple times. The switch lines given by $y(t)=\pi \pm \frac{\pi}{2}$ are also depicted in Fig. 4. Thus, the proposed switched observer has been successfully used in the closed-loop reaction wheel inverted pendulum stabilization.

\section{Conclusion}

We have considered the problem of state estimation for a class of parameter-varying linear systems that are unobservable for certain values of time-varying parameters. This class is given by the system (5), which is a chain of integrators with a known input injection and time-varying sign-changing gains between the integrators. The state estimator is constructed as a switched

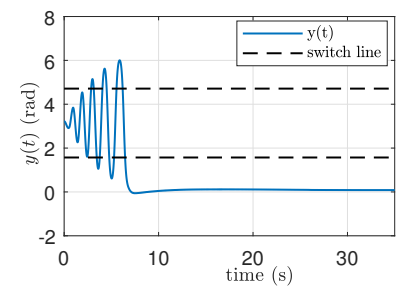

(a) The measured angle $y(t)$.

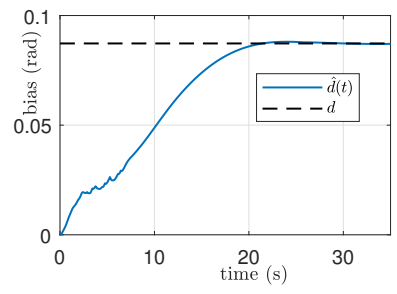

(b) The bias estimate $\hat{d}(t)$.
Fig. 4. Experimental results for the reaction-wheel pendulum system.

observer that commutates when the parameter trajectory travels between predefined subsets, and exponential convergence is shown under a dwell-time assumption on the parameter comportment. Applicability of the proposed solution is illustrated both by simulations and by experimental results for a reaction-wheel pendulum.

For further researches, we intend to consider if the proposed approach can be extended for other classes of observers, such as interval observers and observers with finite/fixed-time convergence.

\section{Acknowledgements}

This work was supported by 111 project No. D17019, China, by Russian Science Foundation grant (project No. 17-79-20341), and by Rennes Metropole, France (project AIS-19C0326).

\section{References}

[1] Angelo Alessandri and Paolo Coletta. Design of luenberger observers for a class of hybrid linear systems. In International workshop on hybrid systems: Computation and control, Rome, Italy, mar. 2001.

[2] Angelo Alessandri and Paolo Coletta. Switching observers for continuous-time and discrete-time linear systems. In American control conference, Arlington, VA, USA, jun. 2001.

[3] Stanislav Aranovskiy, Denis Efimov, Dmitry Sokolov, Jian Wang, Igor Ryadchikov, and Alexey Bobtsov. State estimation for a locally unobservable parameter-varying system: One gradient-based and one switched solutions. In IFAC world congress, Berlin, Germany, aug. 2020.

[4] Karl Johan Åström and Katsuhisa Furuta. Swinging up a pendulum by energy control. Automatica, 36(2):287-295, 2000 .

[5] Georges Bastin and Michel R Gevers. Stable adaptive observers for nonlinear time-varying systems. IEEE Transactions on Automatic Control, 33(7):650-658, 1988.

[6] Francisco Javier Bejarano and Alessandro Pisano. Switched observers for switched linear systems with unknown inputs. IEEE Transactions on Automatic Control, 56(3):681-686, 2011.

[7] Gildas Besançon. Nonlinear observers and applications. Springer Berlin Heidelberg, Berlin, Heidelberg, 2007. 
[8] Daniel J Block, Karl J Åström, and Mark W Spong. The reaction wheel pendulum. Synthesis Lectures on Control and mechatronics, 1(1):1-105, 2007.

[9] Stephen Boyd, Laurent El Ghaoui, Eric Feron, and Venkataramanan Balakrishnan. Linear matrix inequalities in system and control theory. Siam, Philadelphia, PA, 1994.

[10] Henry Cox. On the estimation of state variables and parameters for noisy dynamic systems. IEEE Transactions on Automatic Control, 9(1):5-12, 1964

[11] Denis Efimov, Tarek Raïssi, Stanislav Chebotarev, and Ali Zolghadri. Interval state observer for nonlinear time varying systems. Automatica, 49(1):200-205, 2013.

[12] Mohanarajah Gajamohan, Michael Merz, Igor Thommen, and Raffaello D'Andrea. The cubli: A cube that can jump up and balance. In International conference on intelligent robots and systems, Algarve, Portugal, oct. 2012.

[13] Roger A. Horn and Charles R. Johnson. Matrix analysis. Cambridge University Press, New York, NY, 2012.

[14] Luc Jaulin. Nonlinear bounded-error state estimation of continuous-time systems. Automatica, 38(6):1079-1082, 2002.

[15] Andrew H. Jazwinski. Stochastic processes and filtering theory. Dover Publications, Mineola, NY, 2007.

[16] Frank Jepsen, Anders Soborg, Anders R Pedersen, and Zhenyu Yang. Development and control of an inverted pendulum driven by a reaction wheel. In International conference on mechatronics and automation, Changchun, China, aug. 2009.

[17] Wei Kang, Jean-Pierre Barbot, and Liang Xu. On the observability of nonlinear and switched systems. In Emergent problems in nonlinear systems and control, pages 199-216. Springer, 2009.

[18] Jianxing Liu, Salah Laghrouche, Mohamed Harmouche, and Maxime Wack. Adaptive-gain second-order sliding mode observer design for switching power converters. Control Engineering Practice, 30:124-131, sep. 2014.

[19] Johan Lofberg. YALMIP: A toolbox for modeling and optimization in MATLAB. In International conference on robotics and automation, Barcelona, Spain, apr. 2005.

[20] Henk Nijmeijer and Thor I Fossen. New directions in nonlinear observer design. Springer-Verlag, London, 1999.

[21] Remy Nouailletas, Damien Koenig, and Eduardo Mendes. LMI design of a switched observer with model uncertainty: Application to a hysteresis mechanical system. In Conference on decision and control, New Orleans, LA, USA, dec. 2007.

[22] Stefan Pettersson. Designing switched observers for switched systems using multiple Lyapunov functions and dwell-time switching. In Analysis and design of hybrid systems, Alghero, Italy, jun. 2006.

[23] Héctor Ríos, Denis Efimov, Jaime A Moreno, Wilfrid Perruquetti, and Juan G Rueda-Escobedo. Time-varying parameter identification algorithms: Finite and fixed-time convergence. IEEE Transactions on Automatic Control, 62(7):3671-3678, 2017.

[24] Juan Rueda-Escobedo, Rosane Ushirobira, Denis Efimov, and Jaime Moreno. Gramian-based uniform convergent observer for stable ltv systems with delayed measurements. International Journal of Control, 93(2):226-237, 2020.

[25] Wilson J Rugh. Linear system theory. Prentice Hall, Upper Saddle River, NJ, 1996.

[26] Igor Ryadchikov, Stanislav Aranovskiy, Evgeny Nikulchev, Jian Wang, and Dmitry Sokolov. Differentiator-based velocity observer with sensor bias estimation: An inverted pendulum case study. In IFAC Symposium on nonlinear control systems, Vienna, Austria, sep. 2019.

[27] Hassan Saadaoui, Mohamed Djemaï, Noureddine Manamanni, Thierry Floquet, and Jean-Pierre Barbot. Exact differentiation via sliding mode observer for switched systems. In Analysis and design of hybrid systems, Alghero, Italy, jun. 2006.

[28] Aneel Tanwani, Hyungbo Shim, and Daniel Liberzon. Observability for switched linear systems: Characterization and observer design. IEEE Transactions on Automatic Control, 58(4):891-904, 2013.

[29] Qinghua Zhang. Adaptive observer for multiple-inputmultiple-output (MIMO) linear time-varying systems. IEEE Transactions on Automatic Control, 47(3):525-529, 2002.

[30] Xudong Zhao, Hao Liu, Junfeng Zhang, and Hongyi Li. Multiple-mode observer design for a class of switched linear systems. IEEE Transactions on Automation Science and Engineering, 12(1):272-280, 2015.

\section{A Infeasibility of a solution with a constant ma- trix $P$}

Proposition 7 Consider the matrices $A(y)$ and $C$ given in (3). For any $\delta_{y}, 0<\delta_{y}<1$ there do not exist constant positive definite matrix $P \in \mathbb{R}^{3 \times 3}$ and parameter-varying vector $L: \mathbb{R} \rightarrow \mathbb{R}^{3}$ such that the linear matrix inequality

$$
(A(y)-L(y) C)^{\top} P+P(A(y)-L(y) C)<0
$$

holds for all $y$ such that $|\cos (y)| \geq \delta_{y}$.

PROOF. Assume that for some $\delta_{y}$ the matrices $P$ and $L(y)$ satisfying the LMI (A.1) exist. Since the LMI is satisfied for all $y$ such that $|\cos (y)|>\delta_{y}$, it is also satisfied for $y=0$ and $y=\pi$, that is

$$
\begin{aligned}
& (A(0)-L(0) C)^{\top} P+P(A(0)-L(0) C)<0, \\
& (A(\pi)-L(\pi) C)^{\top} P+P(A(\pi)-L(\pi) C)<0 .
\end{aligned}
$$

Define $A^{\prime}:=A(0)+A(\pi)$ and $L^{\prime}:=L(0)+L(\pi)$. Then

$$
\left(A^{\prime}-L^{\prime} C\right)^{\top} P+P\left(A^{\prime}-L^{\prime} C\right)<0,
$$

implying that the matrix $A^{\prime}-L^{\prime} C$ is Hurwitz. However, due to the structure of the matrices $A^{\prime}=\left[\begin{array}{lll}0 & 2 & 0 \\ 0 & 0 & 0 \\ 0 & 0 & 0\end{array}\right]$ and $C$, the matrix $A^{\prime}-L^{\prime} C$ is singular for any $L^{\prime}$, and the LMI (A.2) is not feasible. Thus, we obtained a contradiction. 